\title{
Antriebssysteme für Stückgutsortieranlagen - eine Bestandsaufnahme
}

\author{
DIPL.-ING. FRANK ABELBECK \\ INSTITUT FÜR TRANSPORT- UND AUTOMATISIERUNGSTECHNIK, LEIBNIZ UNIVERSITÄT HANNOVER
}

\section{Zusammenfassung}

Die Sortierung von Stückgütern stellt einen der aufwendigsten Vorgänge in der Warenverteilung dar, der heutzutage weitestgehend maschinell durch hochautomatisierte, kettengeführte Sortieranlagen erledigt wird. Von besonderem Interesse ist die Ausführung des Antriebssystems, da dessen Eigenschaften maßgeblich die Effizienz der Gesamtanlage festlegen. Berührungslos arbeitende Linearinduktionsantriebe stellen hierfür den aktuellen Stand der Technik dar. Ihr signifikanter Nachteil ist der äußerst geringe Wirkungsgrad von höchstens 25\%. Angesichts steigender Energiepreise wird dieser Nachteil in absehbarer Zeit stärker ins Gewicht fallen. Es müssen Möglichkeiten zur Wirkungsgradsteigerung untersucht werden oder aber ein alternatives, vergleichbares Antriebskonzept gefunden werden. Der Kettenvortrieb durch Reibradantriebe stellt ein solches Konzept dar.

Dieser Beitrag behandelt Antriebssysteme von kettengeführten Stückgutsortieranlagen und stellt zunächst bewährte Systeme wie Kettenrad-, Schleppketten- und Schneckenwellenantrieb vor. Hieran schließt sich eine Diskussion der Eigenschaften von aktuell eingesetzten Linearinduktionsantrieben an. Bezüglich der Wirkungsgradproblematik werden Optimierungsmöglichkeiten aufgezeigt und der Reibradantrieb als weiteres mögliches Antriebskonzept vorgestellt.

\begin{abstract}
Sorting of packaged goods represents one of the most complex and laborious processes of distribution. Current distribution centres rely on highly automated sorting machines composed of a chain of product carriers. The propulsion system of these sorter chains is of interest regarding overall facility efficiency. State-of-the-art drive systems use non-contact, maintenance-free linear inductive motors. A severe disadvantage of this drive concept is its low degree of efficiency of less than $25 \%$. In view of increasing energy costs this disadvantage will become more important in near future. Optimising the efficiency of current linear inductive motors represents one option. Another option is the development of a new drive concept such as chain propulsion by friction wheel drives.
\end{abstract}

This paper addresses drive systems for sorter chains. At first classic systems for propulsion by chain wheel, by dragchain and by worm shaft are reviewed. Subsequently properties of state-of-the-art linear inductive motors as well as optimisation options are discussed. Finally the concept of propulsion by friction wheel drives is introduced.

\section{Einleitung}

Das Sortieren von Stückgut stellt eine Aufgabe der Intralogistik dar, bei der Stückgut von einer oder mehreren Quellen auf eine oder mehrere Senken gemäß vorgegebener Unterscheidungsmerkmale verteilt werden soll [VDI83]. Teilaufgaben sind die Förderung des Stückguts sowie der gesteuerte Wechsel der Fördereinrichtung, das sogenannte Ausschleusen. Bei der Bewältigung dieser Teilaufgaben bieten die kettengeführten Sortieranlagen (Sorter) ein günstiges Verhältnis zwischen technischem Aufwand, Flexibilität und Effizienz. Sie bestehen aus einer endlos geschlossenen, raumgängigen Kette zur Übertragung der Bewegung (Zugorgan) und zur Aufnahme der Last (Tragorgan). Die Ausschleusmechanismen sind an jedem Kettenglied angebracht und laufen mit der Kette um, was als Hauptmerkmal dieser Gruppe von Sortieranlagen angesehen werden kann.

Bekannteste Vertreter sind die Kippschalen- und die Quergurtsorter, benannt nach den jeweils verwendeten Ausschleusmechanismen. Kippschalensorter verwenden schwenkbare Lastaufnahmemittel, deren Kippung den Ausschleusvorgang als Abwurf des Stückguts vollzieht. Quergurtsorter besitzen quer zur Förderrichtung wirkende Fördergurte als Lastaufnahmemittel, eine Ausschleusung geschieht durch Verfahren des Fördergurts. Das Einschleusen erfolgt bei beiden Sortertypen durch geeignete Zuführbänder. 
Kettengeführte Sortieranlagen erzielen aufgrund ihres hohen Automatisierungsgrades und der hohen Verteilgeschwindigkeit erhebliche Zeit- und Kosteneinsparungen, verglichen mit der manuellen Sortierung. Die Eigenschaften des verwendeten Antriebskonzeptes sind dabei maßgeblich für Geschwindigkeit, Zuverlässigkeit und Kosteneffizienz, weshalb nachfolgend wichtige Antriebsprinzipien vorgestellt und diskutiert werden sollen.

Bevor die verschiedenen Antriebssysteme behandelt werden können, ist zunächst das anzutreibende Fördermittel zu betrachten. Es existieren verschiedene Varianten für die Realisierung eines raumgängigen Trag- und Zugorgans [Wil98, S. 8], wobei die heutzutage zumeist verwendete Variante in Abbildung 1 gezeigt ist. Jedes Kettenglied besteht aus einem Zugstab, der per Kugelgelenken mit den benachbarten Kettengliedern verbunden ist. An jedem Zugstab ist eine Traverse befestigt, welche die Trag- und Führungsrollen aufnimmt.

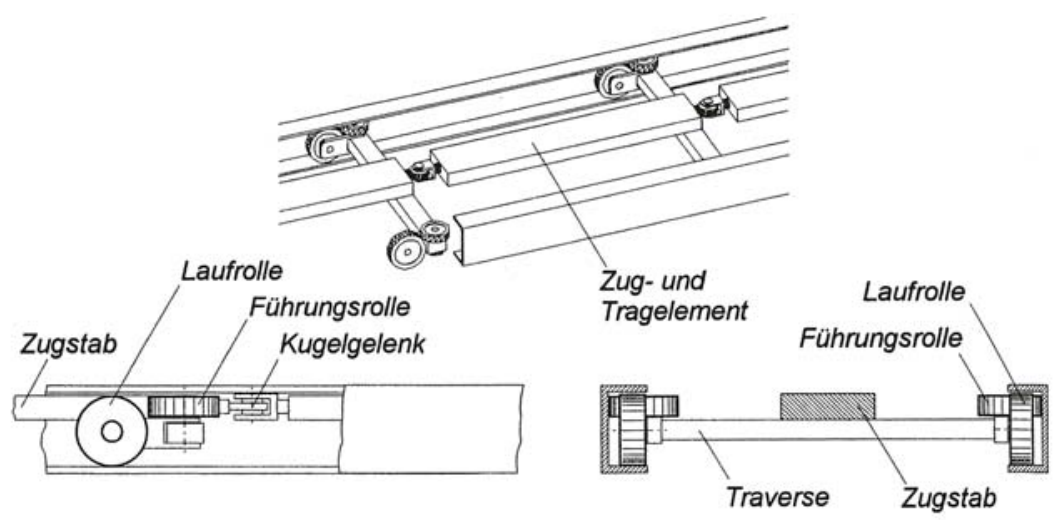

Abbildung 1: Aufbau einer raumgängigen Sorterkette [Wil98, S. 10]

Das Antriebssystem einer Sortieranlage hat die Aufgabe, eine Kraft auf die Kette aufzuprägen, um diese zu beschleunigen, ihre Bewegung gleichmäßig aufrecht zu erhalten oder zu bremsen. Bewährte Konzepte sind die nachfolgend vorgestellten formschlüssigen Antriebe, die die rotatorische Bewegung eines Elektromotors über eine geeignete Mechanik in eine lineare Kettenbewegung umwandeln [Wil98, S. 35 ff.].

\section{Klassische Antriebskonzepte}

\subsection{Kettenradantrieb}

Beim Kettenradantrieb sind in regelmäßigen Abständen senkrechte Mitnehmerbolzen oder -rollen an den Kettengliedern angebracht, auf die durch mehrere große Kettenräder entlang der Anlage formschlüssig die Antriebskraft übertragen wird (Abb. 2). Elastische Materialien dämpfen dabei Stöße infolge des Eingriffs von Rad in Kette.

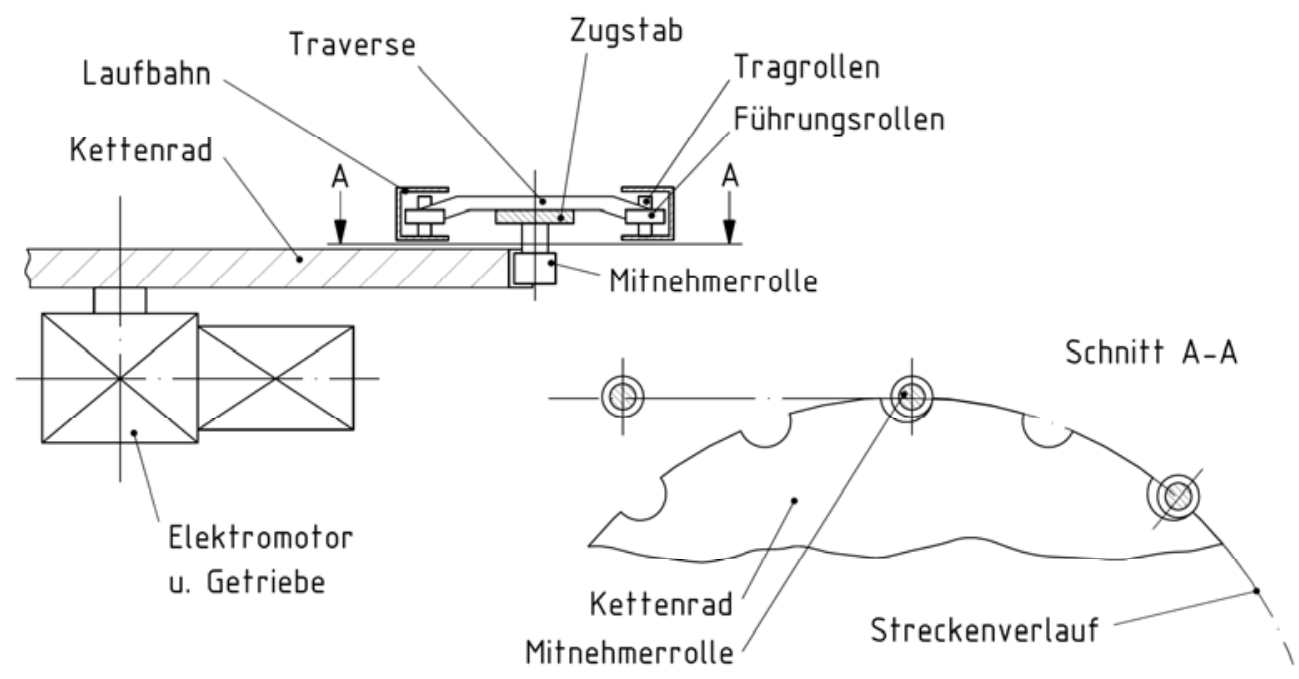

Abbildung 2: Skizze des Kettenradantriebes 


\subsection{Schleppkettenantrieb}

Kettenradantriebe können nur an Umlenkungen der Sorterkette installiert werden. Aus konstruktiver Sicht ist dabei ein kleiner Umlenkradius angestrebt, was bei Sortieranlagen nur bedingt realisiert werden kann. Als Alternative können deshalb anstelle der Kettenräder auch Schleppketten in Form von Buchsen- oder Rollenketten zur formschlüssigen Kraftübertragung eingesetzt werden. Hierzu sind mehrere parallel zur Sorterkette verlaufende Kettentriebeinheiten über die gesamte Anlage verteilt. Die Mitnehmereinrichtungen der Sorterkette werden in die Kette eingefädelt und sodann mitgezogen, wobei der Eingriff wie beim Kettenradantrieb durch Verwendung elastischer Materialien gedämpft werden kann. Im Gegensatz zu den Kettenrädern benötigen diese Antriebseinheiten nur ein kurzes, gerades Streckenstück als Installationsort. Nachteilig ist der erhöhte Geräuschpegel, der weniger durch den - gedämpften - Eingriff in die Sorterkette als vielmehr durch das Laufgeräusch der Schleppkette verursacht wird. Zudem ergibt sich durch den Polygoneffekt der Schleppkette eine Geschwindigkeitsschwankung, die auf die Sorterkette übertragen wird und für Laufunruhe sorgt.

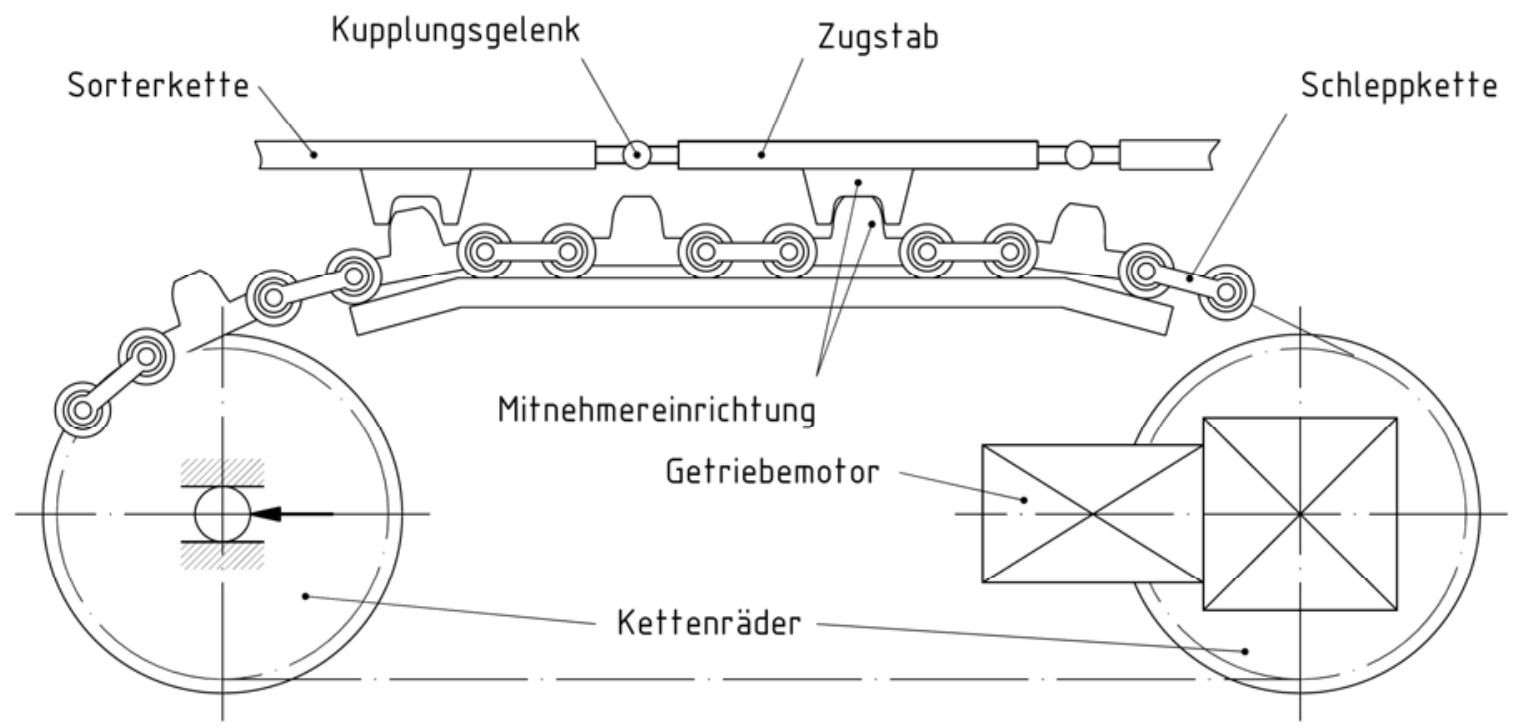

Abbildung 3: Darstellung des Schleppkettenantriebes (vgl. auch [Sal67, S. 270])

\subsection{Schneckenwellenantrieb}

Als weiterer formschlüssiger Antrieb können Schneckenwellen verwendet werden, die ähnlich wie die Schleppketteneinheiten parallel zur Sorterstrecke über die Anlage verteilt installiert werden, aber wie beim Kettenradantrieb leiser und laufruhiger arbeiten. Analog zum Kettenradantrieb sind unter der Sorterkette Mitnehmerrollen angebracht, auf die von den Gewindeflanken der Schneckenwelle eine Antriebskraft aufgebracht wird.

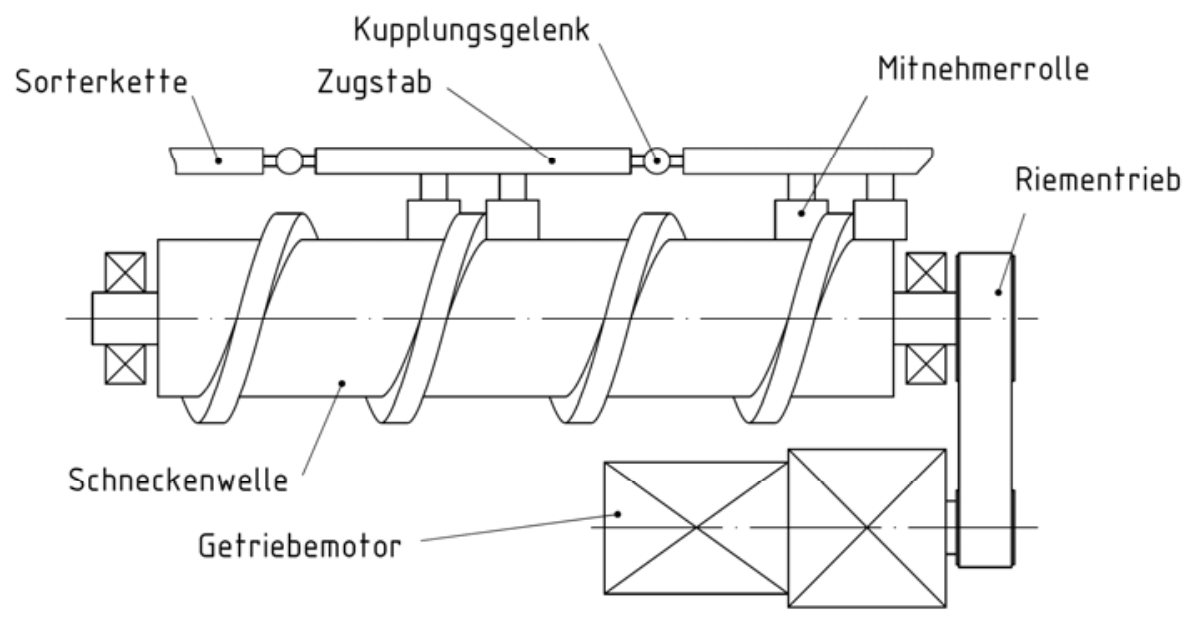

Abbildung 4: Skizze des Schneckenwellenantriebes 


\subsection{Eigenschaften}

Durch die formschlüssige Wirkungsweise ist bei allen genannten Antriebssystemen leicht ein Gesamtwirkungsgrad von ca. 75 \% erreichbar. Nachteilig hinsichtlich Fertigungs- und Montageaufwand ist die für einen formschlüssigen Antrieb genau einzuhaltende Kettenteilung. Andernfalls führen Teilungsdifferenzen zwischen Mitnehmer und antreibender Mechanik zu Laufunruhe und Verschleiß infolge von Flankenstößen beim Eingriff. Da sich dies in der Realität nicht völlig vermeiden lässt, sind diese Systeme entsprechend verschleißbehaftet, weisen Laufunruhe auf und verursachen erhöhte Wartungskosten.

Ein weiterer Nachteil ergibt sich bei Ausfall eines oder mehrerer Antriebe. Denn dann müssen die verbliebenen Antriebe nicht nur den Betrieb aufrecht erhalten, sondern darüber hinaus auch noch den Widerstand der ausgefallenen, aber immer noch im Eingriff befindlichen Antriebssysteme ausgleichen. Dies fällt insbesondere bei den Kettenradantrieben ins Gewicht, denn zumeist ergeben sich nur wenige Installationsorte entlang der Sorterkette. Somit werden Kettenradeinheiten häufig derart ausgelegt, dass eine Einheit die komplette Sorterkette antreiben kann [Wil98, S. 65 ff.].

Beim Übergang zu höheren Fördergeschwindigkeiten von $2 \mathrm{~m} / \mathrm{s}$ und mehr stoßen die Mechaniken schließlich an ihre Grenzen. Naheliegend ist deshalb der Übergang zu einer Antriebsform, die berührungslos direkt eine Kraft in die Kette einzuleiten vermag. Als Folge wurden Linearmotoren als Antriebssysteme eingeführt und stellen aufgrund ihrer Eigenschaften mittlerweile die Standardantriebe für kettengeführte Sortieranlagen dar.

\section{Lineardirektantrieb}

\subsection{Aufbau und Funktionsprinzip}

Eine gängige Methode zur Darstellung von Linearmotoren ist die Umwandlung rotierender elektrischer Maschinen durch Aufschneiden und Abwickeln von Stator und Rotor. Dabei wird der linear angeordnete Stator als Primärteil, der abgewickelte Rotor als Sekundärteil bezeichnet.

Von Bedeutung ist aufgrund der berührungslosen Arbeitsweise die Ausführung als Drehstrommaschinen, von denen sich wiederum der Drehstrom-Asynchron-Linearmotor, auch Linearinduktionsmotor (LIM) genannt, aufgrund seines kostengünstig gestaltbaren Sekundärteils in Form einer einfachen Reaktionsschiene als Sortieranlagenantrieb durchgesetzt hat [Wil98, S. 21]. Hierbei bildet die Sorterkette bzw. daran angebrachte Metallschienen das bewegte Sekundärteil, während mehrere stationäre Primärteile über die Anlage verteilt sind. Abbildung 5 skizziert diese Antriebsart.

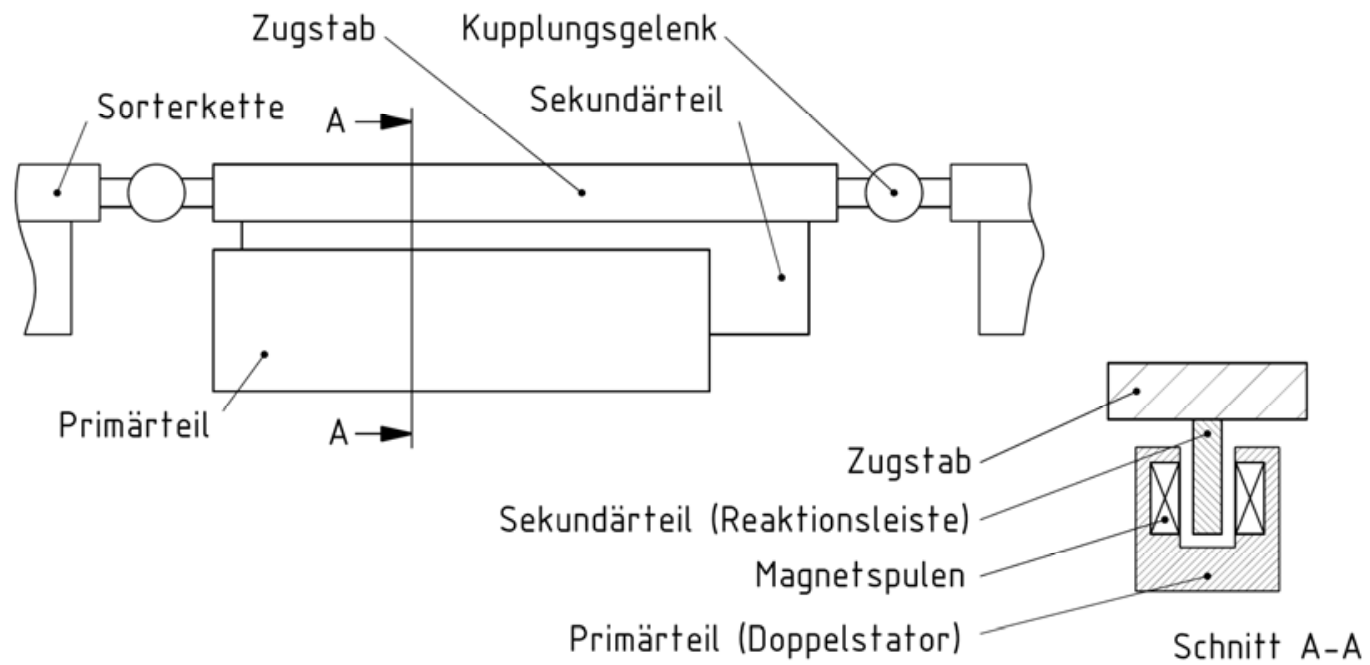

Abbildung 5: Skizze des Antriebes per Linearinduktionsmotor 
Bei der Anordnung der Linearmotorelemente kann ferner in Einfachstator- oder Doppelstatoranordnung unterschieden werden. Die Gestaltung als Doppelstator bietet durch die definierte Position der Primärteile einen konstanten Luftspalt. Beide Primärteile unterstützen sich, wodurch ein stärkeres Magnetfeld aufgebaut werden kann. Und schließlich wird, im Gegensatz zur Einfachstatoranordnung, keine Querkraft auf das Sekundärteil aufgeprägt, wodurch der Scheinleistungsbedarf verringert und der Wirkungsgrad erhöht wird [SEW01]. Dies ist ebenfalls in Abbildung 5 gezeigt.

\section{2. $\quad$ Eigenschaften}

Durch die berührungslose Arbeitsweise stellt der Drehstrom-Asynchron-Linearmotor die gegenwärtig beste Lösung dar, was Instandhaltungsaufwand, Verfügbarkeit und Geräuschverhalten anbelangt. Dabei ist anzumerken, dass bei der Anlagenplanung dem Geräuschverhalten eine sehr große Bedeutung zugewiesen wird und dies ein wesentlicher Grund für die Installation von Linearmotoren ist [Wil98, S. 139]. Aber auch höhere Fördergeschwindigkeiten sind durch den berührungslosen Direktantrieb problemlos realisierbar. Typische verwendete Linearmotoren übertragen eine Kraft von $300 \mathrm{~N}$ bei einer Kettengeschwindigkeit von 2 m/s.

Nachteilig wirken sich die relativ hohen Investitionskosten aus, da Linearmotoren allgemein nur in kleinen Serien produziert werden. Aufgrund ihrer flachen Kraft-Geschwindigkeits-Kennlinie (Abb. 6 links) müssen Linearinduktionsmotoren zudem mit großem Schlupf betrieben werden, um eine befriedigende Motorausnutzung zu erzielen. Die daraus resultierende hohe Relativgeschwindigkeit zwischen Sekundärteil und Drehstromfeld führt zu erhöhten Stromwärmeverlusten im Sekundärteil und zu einem verminderten Wirkungsgrad. Wesentlich stärker wirken sich Verluste infolge des Luftspaltes zwischen Primär- und Sekundärteil aus: Hier bestehen durch den getrennten Aufbau (Primärteil in Fahrbahn, Sekundärteil in Kette) konstruktive Grenzen. Schließlich sind Effekte in den Randzonen des Primärteils, beispielsweise bei Ein- und Ausfahrt des Sekundärteils, für eine weitere Senkung des Wirkungsgrads verantwortlich.
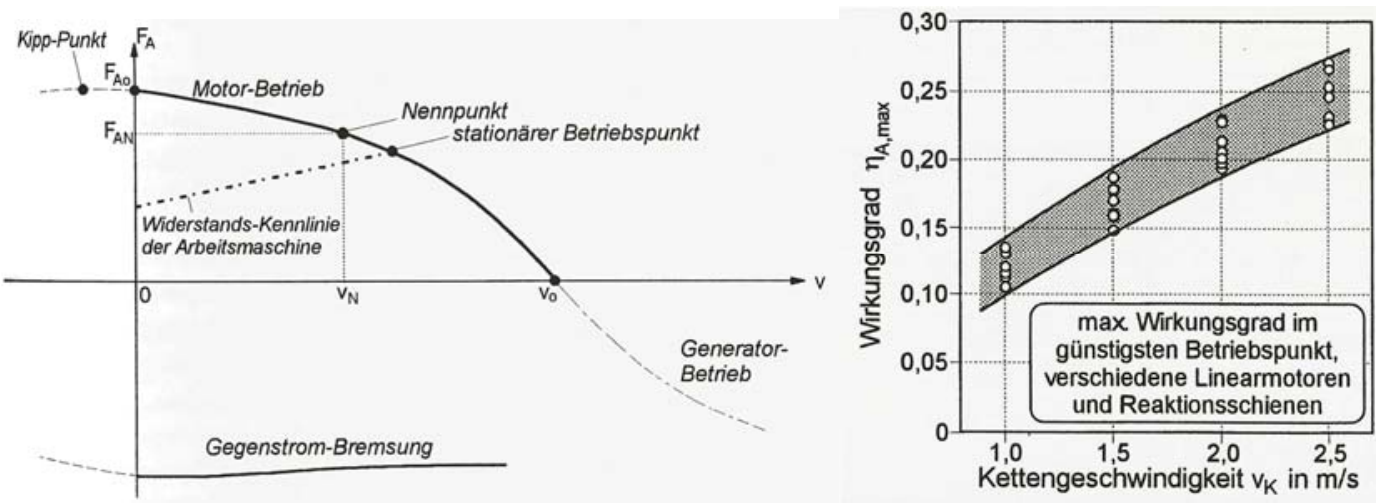

Abbildung 6: Eigenschaften von Linearmotoren; Links: Kraft-Geschwindigkeits-Kennlinie eines DrehstromAsynchron-Linearmotors [Wil98, S. 25]; Rechts: maximaler Wirkungsgrad in Abhängigkeit von der Sorterkettengeschwindigkeit [Wil98, S. 126]

Minimiertes Betriebsgeräusch, Wartungsfreiheit, Laufruhe und hohe Verteilgeschwindigkeit werden beim Antriebssystem Linearmotor somit einerseits durch große Investitionskosten, andererseits durch einen geringen Wirkungsgrad von unter 25 \% erkauft (Abb. 6 rechts).

\subsection{Optimierungsmöglichkeiten}

Vor dem Hintergrund steigender Energiepreise gewinnt eine Verbesserung des Linearmotorwirkungsgrades zunehmend an Bedeutung. Dem kommt einerseits der Trend zu höheren Fördergeschwindigkeiten bei Stückgutsortieranlagen entgegen, da bei Linearinduktionsmotoren eine höhere Kettengeschwindigkeit mit einem höheren Wirkungsgrad einhergeht [Wil98, S. 115/126]. Weiterhin lassen sich verschiedene Parameter optimieren, beispielhaft seien nachfolgend einige genannt.

Material der Reaktionsschiene: Aktuell kommen Aluminiumschienen als Sekundärteil zum Einsatz. Bei Verwendung eines Materials mit höherer Leitfähigkeit kann die Kraft-Geschwindigkeits-Kennlinie steiler gestaltet, der Wirkungsgrad erhöht werden. 
Dicke der Reaktionsschiene: Eine Vergrößerung der Reaktionsschienendicke verringert ebenfalls den Sekundärteilwiderstand, erhöht allerdings den elektrischen Luftspalt und stellt daher einen Kompromiss dar.

Schlitzen der Reaktionsschiene: Durch geeignete Profilierung des Sekundärteils können die Bahnen der induzierten Ströme geeignet geführt werden, was zu einer höheren Antriebskraft bei gleichbleibendem Strombedarf führen kann.

Luftspalt: Durch engere Toleranzen von Primärteil, Fahrschiene und Reaktionsschiene kann der Abstand zwischen Primär- und Sekundärteil verringert werden. Dadurch ergeben sich ein verminderter magnetischer Widerstand und eine geringere Stromaufnahme.

Primärteil: Durch eine Verlängerung der stationären Primärteile können die Längsrandeffekte bekämpft und somit ein Teil der durch Randeffekte hervorgerufenen Verluste reduziert werden.

Das Antriebssystem kann also in Grenzen hinsichtlich des Wirkungsgrades optimiert werden, bleibt dabei allerdings immer noch weit von den Werten der klassischen, formschlüssigen Antriebe entfernt. Weiterhin kommt erschwerend für eine Linearmotoroptimierung hinzu, dass Antriebe von Sorteranlagen selten im optimalen Betriebspunkt, sondern eher im Teillastbereich betrieben werden [Wil98, S. 126]. Zugunsten der überragenden Vorteile wird der Gesichtspunkt der Energieeffizienz zurzeit noch ausgeklammert. Allerdings stellt sich die Frage, ob nicht noch eine Antriebsform denkbar ist, die die Vorteile beider Systeme kombiniert. Eine Antwort darauf ist der Reibradantrieb.

\section{Reibradantrieb}

\subsection{Aufbau und Funktionsprinzip}

Der Reibradantrieb stellt ebenso wie der Linearmotorantrieb einen kraftschlüssigen Antrieb dar, bei dem die Vortriebskraft durch sich gegenüberstehende Reibräder auf an der Sorterkette angebrachte Reibleisten (ähnlich der Sekundärteile beim Linearmotoreinsatz) aufgebracht wird (Abb. 7). Die Reibräder sind in geeigneter Weise an die Reibleiste angepresst und übertragen durch Reibschluss eine von der Anpresskraft und der Materialpaarung abhängige Tangentialkraft auf die Reibleiste. Durch die Verwendung von Standardbauteilen lassen sich die Antriebseinheiten deutlich kostengünstiger fertigen.

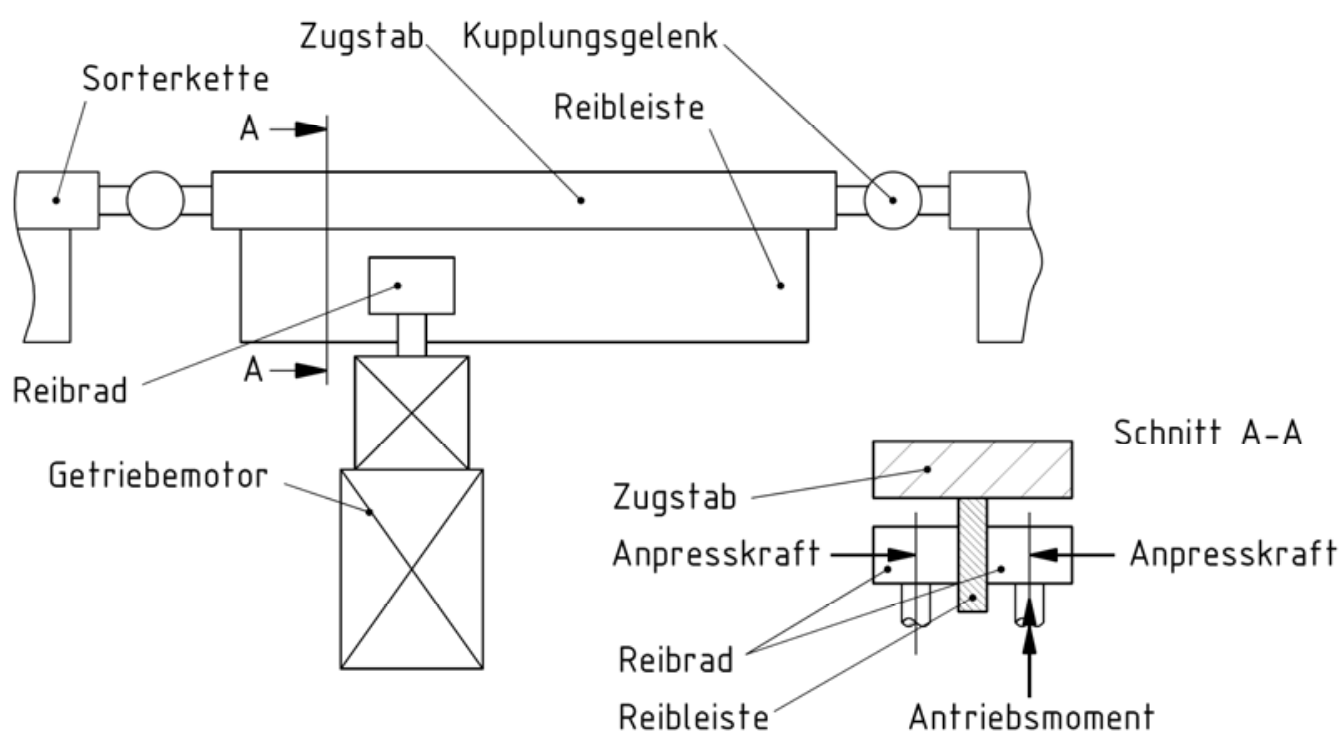

Abbildung 7: Skizze des Reibradantriebes 


\subsection{Eigenschaften}

Vom Wirkungsgrad her ist dieser Antrieb vergleichbar mit den oben genannten formschlüssigen Antrieben, wie durch Messungen von Will seinerzeit am Institut für Fördertechnik in Hannover (heute Institut für Transportund Automatisierungstechnik) bestätigt wurde (Abb. 8). undlagen für die damaligen Untersuchungen lieferte die Arbeit von Bekel, ebenfalls Institut für Fördertechnik, welcher sich Anfang der 1990er Jahre mit der experimentellen Untersuchung von dezentralen Reibradantrieben befasste [Bek92]. Durch die kraftschlüssige Arbeitsweise weist der Reibradantrieb Laufeigenschaften des Linearantriebes auf. So ist bei geeigneter konstruktiver Ausführung ebenfalls ein geräuscharmer und wartungsfreier Betrieb möglich. Dies wurde von Will durch experimentelle Untersuchungen auf einer Versuchsanlage bestätigt. Durch die Ähnlichkeit von Reibleiste und Sekundärteil ist darüber hinaus auch ein kombinierter Betrieb von Reibrad- und Linearantriebseinheiten denkbar, um beispielsweise verschiedene Lastsituationen optimal zu bewältigen.

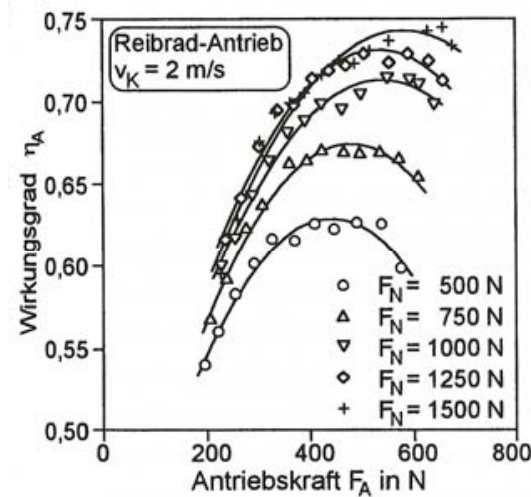

Abbildung 8: Wirkungsgrad des Reibradantriebes, aufgetragen über die Antriebskraft und in Abhängigkeit von der Normalkraft [Wil98, S. 129]

Als Nachteil muss die unterbrochene Reibleiste genannt werden. Ein Reibradantrieb benötigt idealerweise eine ununterbrochene Angriffsfläche. Aufgrund der geforderten Raumgängigkeit der Sorterkette kann dies nicht realisiert werden, insbesondere Vertikalkurven erfordern Spiel zwischen Reibleisten der einzelnen Sorterkettengliedern. Somit treten periodisch stoßartige Beanspruchungen der Reibräder infolge des Einfahrens in die Spalte auf, was wiederum zu einer stoßbehafteten Krafteinleitung führt. Dem kann durch geeignete konstruktive Auslegung der Reibleisten der einzelnen Kettenglieder entgegengewirkt werden. Abbildung 9 zeigt einen Vorschlag von Will, in dem der Spalt zwischen zwei Reibleisten durch Angleichung an den Gelenkweg minimiert wird. Neben dem theoretisch optimalen, kreisförmigen Schnitt wurde auf der Versuchsanlage eine durch gerade Schnitte angenäherte Form verwendet. Experimentelle Untersuchungen von Will zeigten, dass sich die Reibleistenproblematik dadurch hinreichend in den Griff bekommen lässt [Wil98, S. 127].

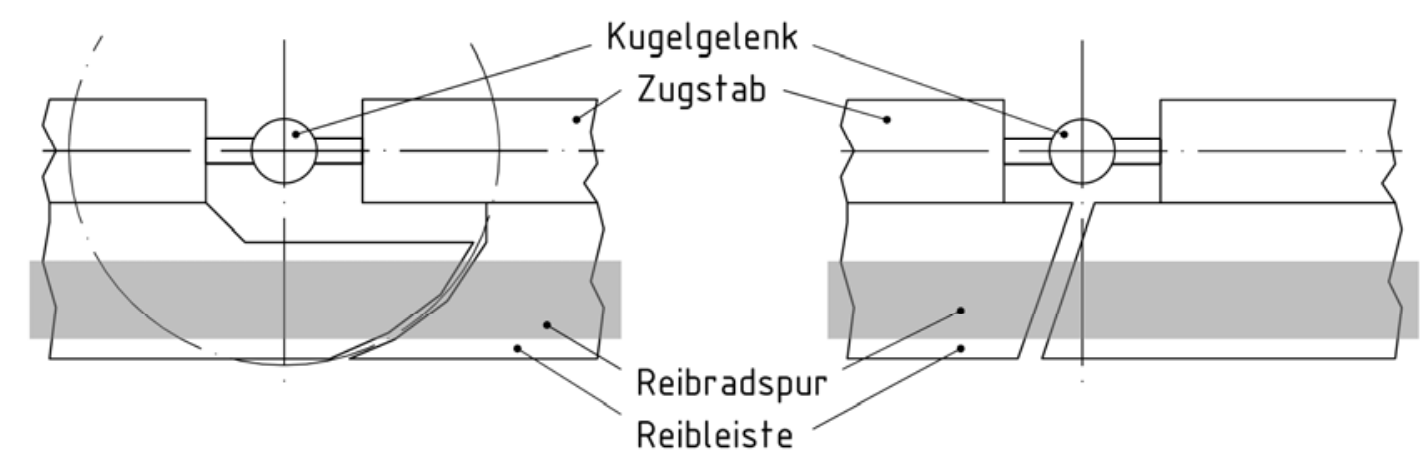

Abbildung 9: Möglichkeiten der konstruktiven Minimierung der Reibleistenspalte: angedeutete Ideallösung und Annäherung durch Schnitte (links), „Green Drive“ (rechts)

Das am Markt verfügbare System „Green Drive“ der Firma Mantissa Corporation setzt ebenfalls auf schräg angeschnittene Reibleisten und breite Reibräder, sodass beim Übergang zwischen zwei Reibleisten immer ein Teil der Reibräder aufliegt [Man02]. Eine weitere Lösung ist die Gestaltung der Reibleiste als durchgängiger, elastischer Gurt, an den die einzelnen Kettenglieder gekoppelt sind [Wil98, S. 50]. 
Dabei entfällt die Kopplung per Kugelgelenk, und es müssen zusätzliche Tragrollen zur Stützung von Nickmomenten der Sorterkettenglieder installiert werden. Ein entsprechendes Patent ist bereits Ende der 1970er eingereicht worden [Can88]. Allerdings wird darin ein relativ breiter Gurt beschrieben, welcher nur bedingt für Vertikalkurven geeignet ist.

Ein weiterer Kritikpunkt ist die Abnutzung der Reibpartner. Im Gegensatz zum Linearmotor funktioniert der Reibradantrieb nicht berührungslos, denn die Kraftübertragung per Wälzreibung ist mit dem Auftreten von Schlupf verbunden. Schlupf wiederum bedeutet Verschleiß, da ein Gleiten der Reibpartner stattfindet und durch kurzzeitige Haftung kleinste Teilchen abgelöst werden können. Prinzipbedingt ist ein Reibradantrieb nicht verschleißfrei, kann aber durch geeignete Auslegung verschleißarm gestaltet werden. Abbildung 10 zeigt den hierbei wichtigen Zusammenhang zwischen tangentialem Kraftschluss (Verhältnis Antriebskraft zu Normalkraft) und Schlupf. Anhand solcher Kurven ist für gegebene Reibpartner der Kraftschluss so zu wählen, dass möglichst wenig Schlupf auftritt.

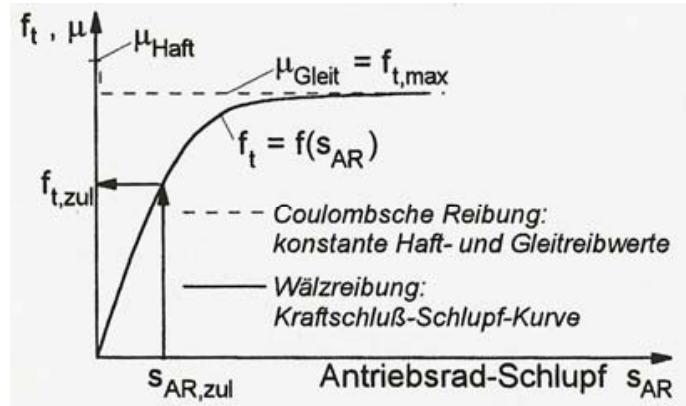

Abbildung 10: Coulombsche Reibung und Wälzreibung im Kraftschluss-Schlupf-Diagramm [Wil98, S. 49]

Wie die Definition des Begriffs Kraftschluss schon andeutet, spielt auch die Anpresskraft bei Verschleißuntersuchungen eine Rolle. Klassische Reibradantriebe verwenden Federn, Gewichte oder Druckschrauben, um eine für den Reibschluss nötige, konstant hohe Normalkraft aufzubringen. Da das Reibradsystem für den ungünstigsten Betriebszustand ausgelegt werden muss, ergibt sich dadurch eine für den Normalbetrieb zu hohe Anpresskraft, die unter anderem zu einem geringeren Wirkungsgrad infolge höherer Radlast führt. Außerdem werden die Komponenten des Antriebs wie beispielsweise die Lager unnötig belastet. Durch geeignete konstruktive Ausführung der Reibradbaugruppe ist es möglich, die Anpresskraft selbsttätig an die zu übertragende Leistung anzupassen [Wil98, S. 47]. Hierzu sind die Reibräder auf Schwingen zu montieren, um die Reaktionskräfte für eine von der Abtriebskraft abhängige Anpresskraft zu nutzen. Abbildung 11 verdeutlicht den Zusammenhang. Diese Lösung ist zwar schon seit Jahrzehnten bekannt (vergl. [TB79, S. 273 f.] mit Bezug auf [OV49]) allerdings, verwendet das Praxisbeispiel „Green Drive“ auch nur eine starre Anstellung über einstellbare Gestänge [Man02, Spalte 16]. Ein Grund dafür ist sicherlich, dass sich diese Art der Anpressung nur für den Betrieb in eine Richtung eignet, für Bewegungsumkehr oder Bremsungen müssten zusätzliche Vorrichtungen angebracht werden.
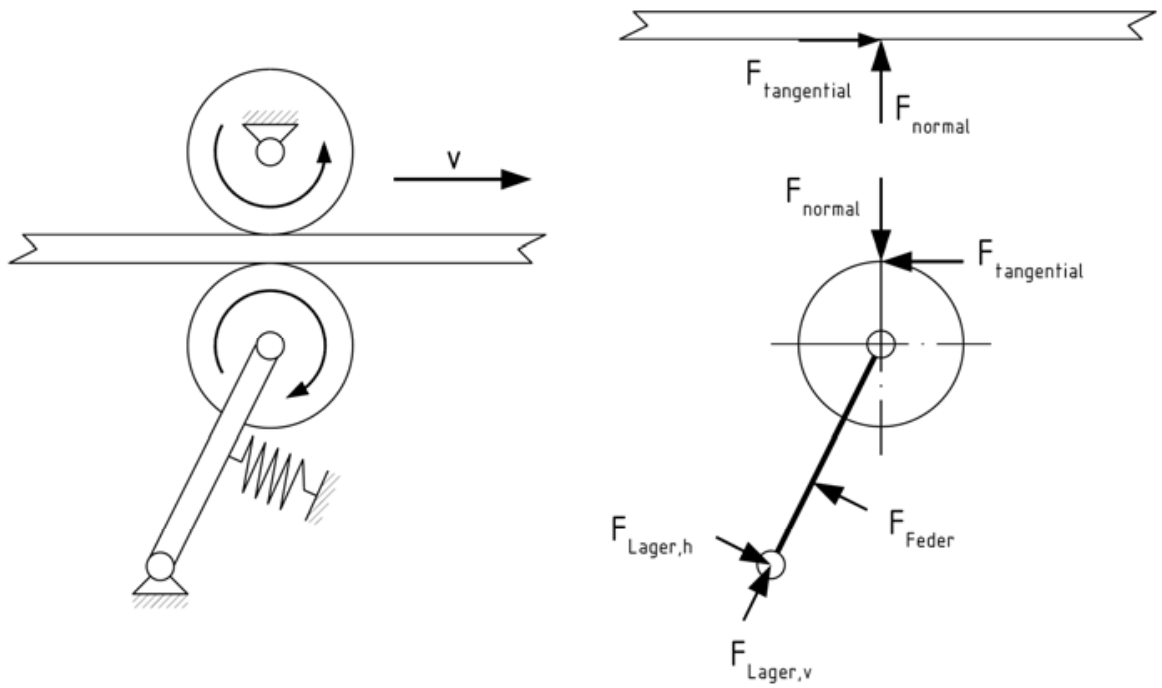

Abbildung 11: lastabhängige Anpressung von Reibrädern 


\section{Zusammenfassung und Ausblick}

Die klassischen, formschlüssigen Antriebe von automatischen Sortieranlagen wurden weitestgehend von wartungsfreien und geräuscharmen Linearmotoren verdrängt. Die genannten Vorteile des Linearmotors werden durch einen sehr niedrigen Wirkungsgrad und hohe Energiekosten erkauft. Es ist anzunehmen, dass dieser zurzeit noch nicht ausschlaggebende Nachteil in Zukunft durch steigende Energiepreise an Relevanz gewinnt. Als Alternative ist der Reibradantrieb anzusehen, der den hohen Wirkungsgrad der formschlüssigen Antriebe mit den guten Betriebseigenschaften des Linearmotors verbindet und parallel zu diesem eingesetzt werden kann.

$\mathrm{Zu}$ beachtende Nachteile sind die unterbrochene Reibleiste sowie die Abnutzung der Reibräder. Ersteres ist durch geeignete Gestaltung der Reibleisten handhabbar, wobei die Arbeit von Will und das System „Green Drive“ die Praxisanwendung demonstrieren. Das Problem der Abnutzung der Reibräder stellt eine Optimierungsaufgabe dar, wobei noch Forschungsbedarf besteht. Dies ist immer noch Gegenstand der Forschung, wie beispielsweise die Arbeiten von Künne und Mehlan zeigen [Meh02]. Schließlich sind konstruktive Lösungen zu finden, um Reibantriebe im Formfaktor der existierenden Linearantriebe anbieten zu können. Dies ist nötig, um einerseits einen einfachen Parallelbetrieb von Linearmotor und Reibradantrieb zu ermöglichen und andererseits die Um- bzw. Aufrüstung bestehender Sortieranlagen zu erleichtern.

Der Antrieb per Reibrad hat das Potential, um den Linearmotor als Antriebssystem von Sortieranlagen zu unterstützen und zu ersetzen. Hierbei sieht das Institut für Transport- und Automatisierungstechnik noch Forschungsbedarf, um den Reibradantrieb fit für den breiten Einsatz in Sortersystemen zu machen.

\section{Literatur}

[Bek92]

[Can88]

[Man02]

[Meh02]

[OV49]

[SEW01]

[TB79]

[VDI83]

[Wil98]
Bekel, S.: Horizontalkurvengängiger Gurtförderer mit dezentralen Reibradantrieben. Fortschritts-Berichte - VDI Reihe 13 Nr. 39. Düsseldorf: VDI-Verlag, 1992. ISBN 3-18-143913-4 Schutzrecht DE 2905313 C2 (21. Januar 1988). Canziani, F. (Erfinder). Pr.: IT 2039678 1978-02-20.

Schutzrecht US 6367610 B1 (9. April 2002). Fortenbery, J. D.; Erceg, D. (Erfinder); Mantissa Corporation (Anmelder).

Mehlan, V.: Experimentelle Verschleißuntersuchungen von angetriebenen Polyurethan-Rädern unter Einwirkung von Zwischenstoffen. Dortmund, Fakultät für Maschinenbau der Universität Dortmund, Diss., 2002.

Opitz, H.; Vieregge, G.: Eigenschaften und Verwendbarkeit von Reibradgetrieben.

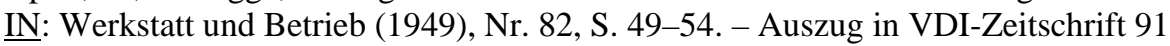
(1949) 575/76

Salzer, G. u. a.: Stetigförderer. Bd. 2. Mainz: KG Krausskopf-Verlag für Wirtschaft GmbH \& Co., 1967.

SEW-EURODRIVE GmbH \& Co. (Hrsg.): Asynchrone Linearmotoren. Katalog 10508406 / DE. Bruchsal: SEW-EURODRIVE GmbH \& Co., 2001.

Tochermann, W.; Bodenstein, F.: Konstruktionselemente des Maschinenbaus: Entwerfen, Gestalten, Berechnen, Anwendungen. Bd. 2: Elemente der drehenden und der gradlinigen Bewegung; Elemente zur Übertragung gleichförmiger Drehbewegungen. 9., verb. Aufl./von F. Bodenstein. Berlin, Heidelberg, New York: SpringerVerlag, 1979. ISBN 3-540-09265-X

VDI-Gesellschaft Materialfluss und Fördertechnik: VDI 3619: Sortiersysteme für Stückgut. Düsseldorf, Mai 1983. - inhaltlich überprüft und unverändert weiterhin gültig: Oktober 1999

Will, F.: Auslegung und Gestaltung von Antriebssystemen für StückgutSortieranlagen. Fortschritts-Berichte - VDI Reihe 13 Nr. 47. Düsseldorf: VDIVerlag, 1998. ISBN 3-18-304713-6 